\title{
Establishment and characterization of histologically and molecularly stable soft tissue sarcoma xenograft models for biological studies and preclinical drug testing
}

Jasmien Cornillie ${ }^{1 *}$, Agnieszka Wozniak ${ }^{1 *}$, Haifu $\mathrm{Li}^{1}$, Yannick Wang ${ }^{1}$, Bram Boeckx ${ }^{2,3}$, Yemarshet K. Gebreyohannes ${ }^{1}$, Jasmien Wellens ${ }^{1}$, Ulla Vanleeuw ${ }^{1}$, Daphne Hompes ${ }^{4}$, Marguerite Stas ${ }^{4}$, Friedl Sinnaeve ${ }^{5}$, Hazem Wafa ${ }^{5}$, Diether Lambrechts ${ }^{2,3}$, Maria DebiecRychter $^{6}$, Raf Sciot ${ }^{7}$ and Patrick Schöffski ${ }^{1}$

${ }^{1}$ Laboratory of Experimental Oncology, Department of Oncology, KU Leuven, and Department of General Medical Oncology, University Hospitals Leuven, Leuven Cancer Institute, Leuven, Belgium ²Laboratory for Translational Genetics, KU Leuven, Leuven, Belgium

${ }^{3}$ VIB Center for Cancer Biology, Leuven, Belgium

${ }^{4}$ Department of Surgical Oncology, KU Leuven and University Hospitals Leuven, Leuven, Belgium ${ }^{5}$ Department of Orthopedic Surgery, University Hospitals Leuven, Leuven, Belgium ${ }^{6}$ Department of Human Genetics, KU Leuven and University Hospitals Leuven, Leuven, Belgium ${ }^{7}$ Department of Pathology, KU Leuven and University Hospitals Leuven, Leuven, Belgium

* both authors contributed equally to this article

\section{Running title}

Mouse xenograft models of soft tissue sarcoma

\section{Keywords}

Soft tissue sarcoma, patient-derived xenograft, NMRI immunodeficient mice, preclinical research model

\section{Additional information}

- Financial support

J. Cornillie received funding from the Bart Verbeeck Fund for Sarcoma Research (KU Leuven, Belgium) and was Emmanuel Vanderschueren Fellow, Kom op tegen Kanker (Stand up to Cancer), the Flemish Cancer Society (Belgium). The experimental work was partially supported by research grants from Fonds voor Wetenschappelijk Onderzoek 
Vlaanderen (FWO grant GA01311N to P. Schöffski) and from Kom op tegen Kanker (Stand up to Cancer), the Flemish Cancer Society (grant to P. Schöffski).

- Corresponding author

Jasmien Cornillie Laboratory of Experimental Oncology and Department of General Medical Oncology University Hospitals Leuven, Leuven Cancer Institute

Herestraat 49

3000 Leuven

Belgium

Phone: +32 16342179

Fax: +32 16346901

jasmien.cornillie@uzleuven.be

- Conflict of interest disclosure statement

The authors declare no potential conflicts of interest.

- Word count abstract: 250 words; word count main text, excluding references: 4643 words Total number of figures: 4

Total number of tables: 2

Total number of supplementary figures: 1

Total number of supplementary tables: 2 


\section{Abstract}

Soft tissue sarcomas (STS) represent a heterogeneous group of rare, malignant tumors of mesenchymal origin. Reliable in vivo sarcoma research models are scarce. We aimed to establish and characterize histologically and molecularly stable patient-derived xenograft (PDX) models from a broad variety of STS subtypes.

A total of 188 fresh tumor samples from consenting patients with localized or advanced STS were transplanted subcutaneously in NMRI nu/nu immunodeficient mice. Once tumor growth was observed, the material was passaged to a next generation of mice. A patient-derived tumor sample was considered "successfully engrafted" whenever the sample was transplanted to passage 1. A PDX model was considered "established" when observing stable morphological and molecular features for at least two passages. With every passage, histological and molecular analyses were performed. Specific genomic alterations and copy number profile were assessed by fluorescence in situ hybridization and low coverage whole genome sequencing.

The tumor engraftment rate was 32\% (61/188) and 188 patient samples generated a total of 32 PDX models, including seven models of myxofibrosarcoma, five dedifferentiated liposarcoma, five leiomyosarcoma, three undifferentiated pleomorphic sarcoma, two malignant peripheral nerve sheet tumor models and single models of synovial sarcoma and some other (ultra-)rare subtypes. Seventeen additional models are in early stages of engraftment (passage 1-2). Histopathological and molecular features were compared with the original donor tumor and were stable throughout passaging. The platform is used for studies on sarcoma biology and suited for in vivo preclinical drug testing as illustrated by a number of completed and ongoing laboratory studies. 


\section{Introduction}

Soft tissue sarcomas (STS) are rare, malignant tumors of mesenchymal origin which account for less than $1 \%$ of all adult malignancies (1). Worldwide, the annual incidence of STS is around $2-3 / 100.000(2,3)$. STS are a histologically, molecularly and genetically very diverse family of malignancies, with more than 70 different subtypes described (4).

Surgery is the mainstay of treatment for localized STS, sometimes supplemented with perioperative radiotherapy or systemic treatment (5). Patients with inoperable or metastatic disease are usually treated with palliative intent, and chemotherapy is the most common treatment approach in this setting. The therapeutic goals in such advanced cases are to gain disease stabilization, to stop or delay progression and achieve or maintain symptom control for as long as possible (2). Five-year overall survival (OS) rates across all STS subtypes and all disease stages are in the range of only $50 \%(3,6)$. Up to $40 \%$ of patients with localized STS develop metastatic spread during their disease course and an additional $10 \%$ present with metastases at the initial diagnosis $(4,7)$. In the majority of cases, metastasis implies that cure can no longer be achieved, with few notable exceptions. Five-year OS in such patients is only around $10-20 \%$ (2). The treatment options for locally advanced, unresectable and/or metastatic disease are far from satisfactory and only a minority of patients achieves objective responses under conventional chemotherapy, which is usually based on doxorubicin monotherapy or on doxorubicin/ifosfamide combination treatment (8). In the most recent randomized Phase 3 trial, the response rate to $75 \mathrm{mg} / \mathrm{m}^{2}$ doxorubicin, which is the standard of care for metastatic or inoperable STS in adult patients, was below $15 \%$ of treated patients (9). After failure of doxorubicin, a variety of chemotherapeutic and targeted agents are used, including ifosfamide, dacarbazine, gemcitabine, trabectedin, eribulin and others (10). Targeted agents play an increasing role in the treatment of STS, with drugs such as the monoclonal antibody olaratumab used in combination with doxorubicin or the small molecule tyrosine kinase inhibitor pazopanib used after failure of anthracycline-based chemotherapy. Although being important and much needed treatment options for patients with advanced STS, all agents used in first and subsequent lines of treatment provide rather limited clinical benefit with progression-free survival typically around only three to six months (11). Therefore, the preclinical or clinical exploration of novel, more effective treatments for STS has high priority. 
Due to the rarity and heterogeneity of sarcomas, most studies pool different subtypes of STS together and include only a relatively small number of patients with specific entities, each subtype characterized by unique disease biology, clinical behavior and response to treatment, as one of the reasons for the somewhat unpredictable outcome of clinical trials in sarcoma. At present, the field is moving towards exploring specific drugs in rationally selected biological entities, as illustrated by large prospective trials performed exclusively in the more common lipo- and leiomyosarcomas $(12,13)$ or by more recent trials in ultra-rare STS variants $(14-17)$. To provide a rationale for such trials there is a need to have reliable sarcoma research models that match the clinical behavior of the according sarcoma subtype and its sensitivity or resistance to a given experimental agent. This facilitates preclinical drug screening and allows to gain better insights into the biology of the disease and to test biology-driven treatment strategies in specific STS subtypes. It enables to screen the expression of specific proteins of interest and preselect the models for preclinical testing based on the expression of targets of interest. This approach gives new agents a higher chance to show their efficacy in the subsequent clinical trials, avoids the high level of uncertainty associated with trials in a nonselected, pooled sarcoma population and can help improving the high attrition rate of drugs in early development for the treatment of STS, that is in part responsible for the slow progression of this scientific field.

During the past decades, cells cultured in vitro and cell line-derived mouse xenografts have been standard tool for preclinical drug screening and development. However, these models are not fully representative for the clinical setting due to the intra- and inter-tumor heterogeneity observed in the clinic (18). Patient-derived xenografts (PDX), established by engrafting human tumor tissue in immunodeficient mouse, can overcome some but not all of these limitations. PDX platforms of different types of cancer have been described, and these PDX models showed to maintain the histological and molecular features of the donor tissue and proved to be a valuable tool in preclinical compound testing (18-22). Moreover, genetically engineered mouse models, generated by selective targeted alteration of a small number of genes, have been developed for a number of STS subtypes (23). These models are useful in cytogenetically simple STS, mostly characterized by tumor-specific chromosomal translocations resulting in fusion genes acting as oncogenic transcription factors. Most STS, however, have a complex karyotype and genetic mouse models have a limited role in the 
preclinical research of these genetically complex sarcomas. Nevertheless, they are very helpful in studying the effects of highly targeted agents.

Due to the rarity of STS there is still an important lack of reliable in vivo research models for this very diverse group of diseases. The aim of our study was to establish and characterize a new, useful panel of PDX models representing different STS subtypes, ranging from common variants to some of the ultra-rare subtypes for which no preclinical models had been available until today.

\section{Materials and methods}

\section{Collection of patient tumor samples and clinical data}

All tumor samples were collected from STS patients, undergoing routine surgery or a diagnostic biopsy at the Departments of Surgical Oncology, Orthopedic Surgery or Radiology, University Hospitals Leuven (Leuven, Belgium). Written informed consent was obtained from each patient and the xenografting of patient-derived mesenchymal tumor material was approved by the Medical Ethics Committee, University Hospitals Leuven (S53483) and by the KU Leuven Ethics Committee for Animal Research (project numbers P062-2011, P184-2012, P056-2014 and P175-2015). We primarily aimed at selecting STS subtypes with specific molecular alterations (e.g. translocation, amplification, point mutation...), allowing easy follow-up and verification throughout passaging. However, as the histological diagnosis was often made only after resection of the specimen, STS subtypes with complex genomic profiles or unknown histological type were also xenografted and subsequently included in the panel. The fresh tumor tissue was collected by the surgeon or radiologist under sterile conditions and a piece of the material was immediately transferred to the Laboratory of Experimental Oncology, KU Leuven in sterile medium. Tumor fragments were transplanted bilaterally into female adult, partially immunodeficient, athymic NMRI nu/nu mice (Janvier Labs). In parallel, tumor tissue was processed by the Department of Pathology, UZ Leuven for routine histology, immunohistochemistry and - if applicable - genetic analysis, e.g. fluorescence in situ hybridization (FISH) or mutational analysis. The diagnosis was made by a reference sarcoma pathologist (RS) in collaboration with an expert sarcoma geneticist (MDR), according to the WHO classification (4). 
To determine potential factors associated with better tumor engraftment, the following donor patient and xenograft sample characteristics were collected: sex, age at diagnosis, origin of the tumor sample (biopsy vs. surgery and primary tumor vs. local relapse vs. metastatic lesion), anatomical location of the sample used for xenografting, histological diagnosis and occurrence of local relapse or metastatic spread during the disease course. These parameters were collected as a part of an extensive LECTOR database, compiling pathological, clinical and molecular characteristic of patients with STS, diagnosed and treated at University Hospitals Leuven, Belgium. These details were compared between successfully engrafted and nonengrafted tumors, using the Fisher's exact and Chi square tests. The Kaplan-Meier method was used to estimate OS of donor patients from successfully engrafted and non-engrafted tumors, which were compared with the log-rank test. $P$ values lower than 0.05 were considered as statistically significant. All statistical calculations were performed with GraphPad Prism version 7 software (GraphPad Software Inc).

\section{Establishment of STS PDX models}

Tumor samples (25-75 $\mathrm{mm}^{3}$, depending on the availability of tissue) were transplanted bilaterally into the subcutaneous space of immunosuppressed mice (passage 0), under $3 \%$ isoflurane anesthesia (Rotacher). Mice were housed in individually ventilated cages, with food and water available ad libitum, and the tumor growth was checked weekly. Once tumor growth was observed and its volume reached approximately $200 \mathrm{~mm}^{3}$, the tumor-bearing mouse was euthanized using a supraphysiological dose of pentobarbital, and the tumor was bilaterally re-transplanted ("passaged") to a next generation of mice $(n=2)$. With every passage, a piece of tissue was snap-frozen in liquid nitrogen and another piece was fixed in $4 \%$ buffered formalin to assess the histopathological and molecular features of the tumor.

A xenograft sample was considered "successfully engrafted" whenever a growing tumor could be transplanted to a next generation of mice (passage 1 or higher). A xenograft model was considered "established" after observing stable histological and molecular features for at least two passages (retained characteristics in passages 1 and 2, identical to those of the original patient's tumor).

Xenograft tumor pieces from established models were also collected in $10 \%$ dimethyl sulfoxide (DMSO; Sigma-Aldrich) in Dulbecco's Modified Eagle Medium Nutrient Mixture F-12 (DMEM/F-12; Thermo Fisher Scientific) for long-term cryopreservation, i.e. storage in liquid 
nitrogen. This allowed for biobanking of PDX material, which can be used at a later time point to re-induce the PDX model in mice for in vivo experiments. Cryopreserved material was retransplanted into mice after at least 6 months of storage in liquid nitrogen and in vivo regrowth of the PDX material was assessed. After ensuring the regrowth of the tumor model and after collection of sufficient ex-mouse material, fast-growing models were discontinued in mice, with the possibility to re-induce the PDX model in vivo at a later time point.

For ethical reasons, we chose to engraft tumors bilaterally in order to reduce the number of mice required. In order to ensure that two tumors from the same animal can be considered as independent events we used previously obtained datasets (24). They included tumor volume and mitotic/apoptotic count, from three different in vivo experiments performed in three different PDX models, randomizing (a) control mice, (b) mice treated with drug A and (c) mice treated with drug $B$, with at least 6 mice (12 tumors) included in each treatment group. Using these data we calculated the degree of similarity or clustering between two tumors engrafted in the same mouse by quantifying the intra-class correlation coefficient (ICC), ranging from 0 (no similarity) to 1 (similar data). The ICCs were obtained from a one-way random effects model using the procedure PROC MIXED in the SAS software, version 9.4 of the SAS System for Windows (25).

\section{Histopathological and molecular characterization of STS PDX models}

Formalin-fixed tumor specimens were embedded in paraffin and $4 \mu \mathrm{m}$ sections were cut for hematoxylin and eosin (H\&E) staining and immunohistochemical analyses. The following primary antibodies were used for immunohistochemistry: alpha smooth muscle actin ( $\alpha$-SMA; Dako M085129-2), desmin (Dako M076001-2), epithelial membrane antigen (EMA; Dako M061329), myogenin (Dako M3559), S100 (Dako A511401-8), murine double minute 2 homolog (MDM2; Thermo Fisher Scientific 337100) and human leukocyte antigen A (HLA-A; Abcam ab52922). Sections were incubated with secondary antibody-HRP polymer conjugate (Envision+ System-HRP; DAKO) and stainings were developed using diaminobenzidine (DAB; DAKO), followed by hematoxylin counterstaining (VWR).

A dual-color FISH was conducted on paraffin sections to assess STS-specific genetic changes: MDM2 amplification, rearrangements of synovial sarcoma translocation chromosome 18 (SS18), calmodulin binding transcription activator 1 (CAMTA1) and capicua transcriptional repressor $(C I C)$ and the fusion between hes-related family bHLH transcription factor with 
YRPW motif 1 (HEY1) and nuclear receptor coactivator 2 (NCOA2). The LSI MDM2/CEP 12 FISH Probe Kit (Kreatech Diagnostics KBI-10717) and Vysis SS18 Break Apart FISH Probe Kit (Abbott 03N61-020) were used to detect MDM2 amplification and SS18 rearrangement, respectively. For assessment of CAMTA1 and CIC rearrangement and HEY1-NCOA2 fusion, home-made FISH probe sets designed, labelled and validated in the Department of Human Genetics, KU Leuven were used.

\section{Low coverage whole genome sequencing}

In order to study the genomic stability between passages, copy number analysis was performed using low coverage whole genome sequencing (coverage $0.1 \mathrm{x}$ ). DNA was extracted from frozen xenograft samples of different passages, using the QIAamp DNA Mini Kit (Qiagen). KAPA DNA Library Preparation Kit (Illumina) was used to prepare DNA libraries, which were sequenced at low coverage on a HiSeq2000 (Illumina) with a V3 flowcell generating 50bp reads. Raw reads were aligned to the human reference genome version hg19 (https://genome.ucsc.edu; hg19 released Feb 2009) with Burrows-Wheeler Aligner software package (http://bio-bwa.sourceforge.net) and after duplicate removal further analyzed with QDNAseq (https://bioconductor.org/biocLite.R) to exclude known regions with low mapping quality, correct for the genomic wave and to count the reads per bin. Binned data were further segmented with the ASCAT (Allele-Specific Copy number Analysis of Tumours) algorithm (https://www.crick.ac.uk/peter-van-loo/software/ASCAT). The library preparation, sequencing and data analysis was performed in the Laboratory of Translational Genetics, Department of Human Genetics and Vesalius Research Center, KU Leuven and Flemish Institute of Biotechnology (Vlaams Instituut voor Biotechnologie - VIB).

\section{Results}

\section{Patient characteristics and tumor engraftment}

From September 2011 until May 2018, 188 clinical STS samples were obtained from consenting patients during routine surgery or biopsy procedures and were transplanted into mice. The samples were collected from 188 individual patients, with a male-to-female ratio of 1.2 and a median age at diagnosis of 61 years (range 18 -92). Eighty-three percent of the samples were collected during surgical excision of tumor tissue, while the remaining $17 \%$ were obtained in the context of a diagnostic biopsy (needle, endoscopic or surgical biopsy or cytology). The donor tissue was most often collected from a primary tumor (68\%), followed 
by local recurrence $(19 \%)$ or metastatic lesion (13\%). The transplanted tissue was resected or biopsied from extremities in the majority of cases (47\%), but also from the abdominal compartment $(24 \%)$, trunk (16\%), pelvis $(12 \%)$ or the head and neck region (1\%). The most common STS subtypes transplanted were dedifferentiated liposarcoma (DDLPS) (16\%), leiomyosarcoma (14\%), myxofibrosarcoma (11\%), myxoid liposarcoma (7\%), atypical lipomatous tumor (ALT) (5\%), undifferentiated pleomorphic sarcoma (5\%), synovial sarcoma (4\%), undifferentiated sarcoma not otherwise specified (4\%), malignant peripheral nerve sheath tumor (MPNST), solitary fibrous tumor (SFT), undifferentiated spindle cell sarcoma, extraskeletal myxoid chondrosarcoma and angiosarcoma (each 3\%). The remaining samples were derived from a diverse group of (often ultra-rare) STS subtypes, and were grouped together as 'other'. Characteristics of donor patients and xenografted tissue and the corresponding in vivo engraftment rates are summarized in Table 1 and 2.

Seventy-seven of the 188 donor patients (41\%) developed metastases at any time during their course of disease (either before or after the surgery/biopsy). We did not observe a statistically significant difference in tumor engraftment rate of samples collected from such patients in comparison with specimens from individuals who did not develop metastases ( $40 \%$ and $27 \%$ respectively). However, patients whose tumor successfully engrafted in mice had significantly poorer OS than those whose tumor did not engraft, with a median OS of 77 months compared to 259 months ( $p=0.02$, Figure 1). Patients' sex and age at diagnosis did not influence the engraftment of tumors in our immunodeficient mice. Furthermore, no significant differences in success rate were observed between surgical specimens and diagnostic biopsies or between primary tumors, recurrent tumors and metastatic lesions. Anatomical location or histological subtype of the transplanted tissue also did not have an impact on the engraftment rate. There was no difference in engraftment success between patients who had a local relapse throughout the course of their disease and those who did not develop recurrent disease (Table 1).

\section{Panel of PDX models of STS (XenoSarc)}

In total, we have established 32 PDX models, including seven models of myxofibrosarcoma, five DDLPS, five leiomyosarcoma, three undifferentiated pleomorphic sarcoma, two MPNST models and single models of synovial sarcoma, epithelioid hemangioendothelioma, pleomorphic rhabdomyosarcoma, pulmonary artery intimal sarcoma, mesenchymal 
chondrosarcoma, CIC-rearranged round cell sarcoma, myxoinflammatory fibroblastic sarcoma, rhabdomyosarcoma not otherwise specified, telangiectatic extraskeletal osteosarcoma and undifferentiated sarcoma not otherwise specified (Table 2). A detailed overview of the established PDX models, including current passage, growth rate, histological and molecular features and treatment history of the donor patient, can be found in Supplementary Table S1. An additional 17 models are currently in early stages of engraftment (passage 1 and 2).

Human origin of the ex-mouse STS tumor tissue was confirmed by positive HLA-A immunostaining. Comparison of the H\&E-stained patient material and the corresponding PDX models showed similar morphology, which remained preserved throughout passaging. Figure 2 shows representative $H \& E$ images of donor tissue and the last available passage of the corresponding STS PDX model. In addition, xenografted and original patient tumors showed similar patterns of expression of characteristic immunohistochemical markers (Figure 3, Supplementary Table S1).

For STS subtypes that are characterized by a specific molecular marker the presence of the according genetic hallmark was confirmed in the PDX model using FISH (Supplementary Table S1). In all DDLPS models (UZLX-STS3, -STS5, -STS112, -STS124, and -STS158), MDM2 (12q15) amplification was present throughout passages (Figure 4A, B). Specific translocations were confirmed using a split-apart approach of SS18 in the UZLX-STS7 synovial sarcoma model (Figure 4C), CAMTA1 in the UZLX-STS15 epithelioid hemangioendothelioma model (Figure 4D) and $\mathrm{CIC}$ rearrangement in the UZLX-STS134 CIC-rearranged round cell sarcoma model (Figure 4E). Fusion between HEY1-NCOA2 was confirmed in the UZLX-STS41 mesenchymal chondrosarcoma PDX model (Figure 4F).

Genomic profiles obtained through low coverage whole genome sequencing, describing copy number gains and losses in different passages from the same PDX model, were compared. Paired xenografted material was available from 17 out of 32 STS PDX models; genomic profiling data from original patient material was available from the MPNST model UZLX-STS39 and from the mesenchymal chondrosarcoma model UZLX-STS41. Although most profiles were very complex, we observed stable copy number profiles throughout passaging as exemplified in Supplementary Figure S1. DNA sequencing data have been deposited in the ArrayExpress database at EMBL-EBI (www.ebi.ac.uk/arrayexpress) under accession number E-MTAB-7826. 
From several established models, we used ex-mouse material that had been cryopreserved over a period of at least 6 months to re-engraft in mice. We were able to re-introduce 25 out of 27 PDX models from which material was stored in liquid nitrogen for at least 6 months. The corresponding PDX models were successfully regrown in mice, except for the leiomyosarcoma model UZLX-STS81 and the undifferentiated pleomorphic sarcoma model UZLX-STS104. After these experiments, 11 of fast-growing PDX models were temporarily discontinued in mice after collection of sufficient cryopreserved PDX material (Supplementary Table S1). All other models are continuously kept in animals.

\section{Validation of bilateral PDX engraftment}

The XenoSarc panel of STS xenografts is characterized by stable histological and molecular features, reflecting the phenotype and genotype of the original patient samples, and is therefore well suited for in vivo drug testing of innovative agents. To test the hypothesis that two tumors, engrafted on the left and right flank of the mouse, can be considered as independent events, the ICC was quantified, using data obtained previously (i.e. tumor volume, mitotic and apoptotic activity) (24). In eight out of nine analyses, calculated ICC values were below 0.4 , suggesting a poor correlation between left and right tumors from a single animal. This implies that data simplification, by ignoring the correlation between two tumors from the same mouse, will have a negligible impact on the statistical analyses and the drawn conclusions. Therefore, we decided to consider two tumors from the same animal as independent events. ICCs for tumor volume, mitotic and apoptotic activity of the three studied datasets are shown in Supplementary Table S2. These results ensure that the approach to engraft tumors bilaterally produces reliable results while the number of mice required for the experiment is reduced.

\section{Discussion}

Over a period of 93 months, we were able to collect and transplant material from 188 STS patients, which resulted in the establishment of 32 well-characterized PDX models (XenoSarc platform). This work was based on our xenograft expertise built between 2004 and 2010, a period during which we established a number of mouse models from gastrointestinal stromal tumor, the most common STS of the gastrointestinal tract (26-28). Our current expanded platform covers models from diverse STS subtypes including some ultra-rare entities, all 
replicating the histological and molecular features of the donor tissue and maintaining the characteristics throughout passaging. Although copy number alterations have been reported to accumulate rapidly in PDX (29), copy number profiles in our PDX models were, although sometimes complex, highly stable throughout passaging, which is a very reassuring finding in our series.

To our knowledge, the established STS PDX platform represents the largest and most diverse collection of STS PDX models established by a non-commercial institution till date. Previously, other groups had established stable PDX models of myxoid liposarcoma $(n=7)$, uterine leiomyosarcoma $(n=10), \operatorname{MPNST}(n=5), \operatorname{SFT}(n=2)$, undifferentiated pleomorphic sarcoma $(n=1)$, alveolar rhabdomyosarcoma $(n=1)$, embryonal rhabdomyosarcoma $(n=1)$ and CIC-DUX4 sarcoma $(n=1)(30-36)$. However, this is the first report on the establishment of several other STS PDX subtypes using identical methodology and infrastructure, including some common subtypes such as myxofibrosarcoma and synovial sarcoma, but also some ultra-rare entities such as epithelioid hemangioendothelioma, pulmonary artery intimal sarcoma and mesenchymal chondrosarcoma. Of note, these models are accompanied by extensive clinical annotation (LECTOR database) and patients' samples, including tumor material and blood samples from donor patients.

A total of 188 fresh, surgically resected or biopsied tumor samples from consenting patients with localized or advanced STS were transplanted in mice, with leiomyosarcoma and DDLPS being the most common STS subtypes in our patient cohort. These STS subtypes were also identified as the most prevalent subtypes in a large population-based study including 1463 patients with STS (37), underlining the representativeness of our patients diagnosed and treated in University Hospitals Leuven and participating in the voluntary donation of tissue for research purposes. With 61 out of 188 samples successfully growing in mice, the overall tumor engraftment rate was $32 \%$. In the literature, engraftment success rates up to $76 \%$ have been reported when generating subcutaneous PDX models derived from patients with advanced STS (38). Several factors might explain the lower engraftment rate observed in our study. First of all, the STS samples transplanted in this study were derived from a mixed patient population, including patients with both localized and advanced disease. We, however, did not observe a significantly higher engraftment rate when transplanting samples from patients who developed metastatic disease compared to those with localized disease $(40 \%$ vs. $27 \%$ 
respectively). We also did not observe higher engraftment rate either when transplanting metastatic lesions. However, metastatic lesions were mostly biopsied and not resected, and the smaller volume of tumor tissue obtained through biopsy is known to reduce the chance of successful engraftment (39). When considering the reported engraftment rate, other methodological aspects also have to be taken into account. In order to limit the number of mice, we transplanted the human-derived tissue to a single mouse (passage 0), while other groups transplanted the patient material to a group of mice, hereby increasing the changes to successful engraftment. Moreover, as high-grade STS tumors are often characterized by extensive necrosis (40), the transplantation of a single, small tumor piece increases the possibility for sampling error and transplantation of necrotic tissue, which will not engraft. Additionally, more severely immunosuppressed mouse strains such as severe combined immunodeficient (SCID) mice are assumed to be better suited for xenoengraftment, although this was not confirmed in a recently published study that established uterine sarcoma and carcinosarcoma PDX models (31), while using SCID mice requires a more advanced animalium set up and increases the cost of the establishment and maintenance of such PDX platform.

Recently, there has been some controversy concerning the genomic stability of PDX models. Ben-David et al. studied the copy number profile of 543 PDX models across different tumor types, including 45 STS PDX models. They identified rapid accumulation of copy number alterations during passaging (29). These results are in contrast with various previously published reports, in which a remarkable concordance was shown between the copy number alterations of the donor tissue and the corresponding PDX model (41), which was also confirmed in our study. Several reasons might explain this discrepancy. First of all, PDX models might carry the genomic rearrangement intrinsic to tumor progression. This was suggested by a study of Ding et al., in which a basal-like breast cancer PDX model displayed a mutation enrichment that was also observed in brain metastases derived from the same patient (42). A study with sarcoma PDX models also showed that many of the copy number alterations observed in the models were frequently observed in patients with sarcoma (43). Moreover, the difference in expression profile is thought to be partly a result of the loss of human stroma in PDX models throughout passaging. In this respect, the higher frequency of copy number alterations throughout passaging can be explained by the increasing tumor DNA purity in the PDX model, avoiding cross-contamination by normal DNA of the human stroma (41). 
Furthermore, orthotopic instead of subcutaneous xenografting of tumor tissue has been shown to more reliably mimic the biology of the human tumor because of the maintenance of the original tumor micro-environment and organ-specific pathophysiology (44). However, subcutaneous implantation of tumor tissue might more closely resemble the orthotopic tissue environment in STS compared to epithelial-derived carcinomas. In our analysis, copy number profiles of PDX models were, although sometimes complex, highly stable throughout passaging, confirming the fidelity of PDX models for preclinical cancer research. However, being aware of the genotypic changes that tumors can undergo throughout passaging, it is highly recommended to perform genomic profiling of the PDX samples with every 1-2 passages.

All the established PDX STS models are available for biological studies, preclinical compound testing and other relevant experiments. Most models are kept in vivo through consecutive passaging although some fast-growing models have been cryopreserved in order to reduce the number of animals needed over time. We confirmed successful re-engraftment of the cryopreserved tissue from all these PDX models in mice. The concept of cryopreservation for storage of PDX models and subsequently thawing and re-implanting of tumor tissue is an established method for efficient PDX biobanking (45), but a relatively new experience for our laboratory.

Apart from histological characterization, we performed RNA-sequencing on the established PDX models to identify genetic alterations in cancer-related genes and we have constructed tissue microarrays of the available PDX models, enabling the rapid screening of specific markers of interest throughout the different PDX models. In conclusion, the detailed characterization of the PDX models, reflecting the pheno- and genotype of the original patient samples, facilitates the rational selection of appropriate PDX models for future drug experiments. This experimental approach could reduce the high failure rate observed in early clinical trials in STS, which are mainly purely empirical clinical trials, without preselecting the experimental drug or the patient cohort based on biological or molecular grounds. The availability of PDX models of some unique, ultra-rare entities allows us to study the biology of these orphan diseases and subsequently test biology-driven therapies in these unique PDX models. The utility of PDX models as a preclinical platform for assessment of drug efficacy was recently confirmed by a comprehensive study of Izumchenko et al., showing a significant 
association between drug responses in patients and their corresponding PDX models in 112 of the 129 investigated cases (46). Some of our established PDX models have already been successfully used for in vivo testing of novel agents including tyrosine kinase inhibitors and cytotoxic prodrugs $(24,47)$. Over the course of time we aim to perform multiple additional preclinical efficacy studies using our PDX platform. The models are also accessible for collaborative studies with academic or commercial partners. Hopefully, the results obtained from this preclinical research will enable the sarcoma research community to prioritize suitable treatment strategies to be tested in clinical trials, with the ultimate goal to improve patient care in the field of STS.

\section{Acknowledgements}

We gratefully acknowledge the contribution of the late Dr. Ignace Samson to the collection of tissue for this project. We want to thank Lise Vreys for her technical support. 


\section{References}

1. Clark MA, Fisher C, Judson I, Thomas JM. Soft-tissue sarcomas in adults. N Engl J Med 2005;18:701-11

2. Schöffski P, Cornillie J, Wozniak A, Li H, Hompes D. Soft tissue sarcoma: an update on systemic treatment options for patients with advanced disease. Oncol Res Treat 2014;37:355-62

3. Stiller CA, Trama A, Serraino D, Rossi S, Navarro C, Chirlaque MD et al. Descriptive epidemiology of sarcomas in Europe: report from the RARECARE project. Eur J Cancer 2013;49:684-95

4. Fletcher CD, Bridge JA, Hogendoorn PC, Mertens F. WHO classification of tumours of soft tissue and bone. Lyon: IARC Press; 2013

5. ESMO/European Sarcoma Network Working Group. Soft tissue and visceral sarcomas: ESMO Clinical Practice Guidelines for diagnosis, treatment and follow-up. Ann Oncol 2014;25:iii102-12

6. Ries LA, Young JL, Keel GE, Eisner MP, Lin YD, Horner MJ. SEER Survival Monograph: cancer survival among adults: U.S. SEER program, 1988-2001, patient and tumor characteristics. National Cancer Institute, SEER Program, NIH Pub No. 07-6215, Bethesda, 2007

7. Coindre JM, Terrier P, Guillou L, Le Doussal V, Collin F, Ranchère D et al. Predictive value of grade for metastasis development in the main histologic types of adult soft tissue sarcomas. Cancer 2001;91:1914-26

8. Bramwell V, Anderson D, Charette M, Sarcoma Disease Site Group. Doxorubicin-based chemotherapy for the palliative treatment of adult patients with locally advanced or metastatic soft tissue sarcoma. Cochrane Database Syst Rev 2013;3:CD003293

9. Judson I, Verweij J, Gelderblom H, Hartmann JT, Schöffski P, Blay JY et al. Doxorubicin alone versus intensified doxorubicin plus ifosfamide for first-line treatment of advanced or metastatic soft-tissue sarcoma: a randomised controlled phase 3 trial. Lancet Oncol 2014;15:415-23

10. Lee AT, Pollack SM, Huang P, Jones RL. Phase III soft tissue sarcoma trials: success or failure? Curr Treat Options Oncol 2017;18:19;doi:10.1007/s11864-017-0457-1

11. Ratan R, Patel SR. Chemotherapy for soft tissue sarcoma. Cancer 2016;122:2952-60.

12. Demetri GD, von Mehren M, Jones RL, Hensley ML, Schuetze SM, Staddon A et al. Efficacy and Safety of Trabectedin or Dacarbazine for Metastatic Liposarcoma or Leiomyosarcoma After Failure of Conventional Chemotherapy: Results of a Phase III Randomized Multicenter Clinical Trial. J Clin Oncol 2016;34:786-93

13. Schöffski P, Chawla S, Maki RG, Italiano A, Gelderblom H, Choy E, Grignani G et al. Eribulin versus dacarbazine in previously treated patients with advanced liposarcoma or leiomyosarcoma: a randomised, open-label, multicentre, phase 3 trial. Lancet 2016;387:1629-37

14. Judson I, Scurr M, Gardner K, Barquin E, Marotti M, Collins B, et al. Phase II study of cediranib in patients with advanced gastrointestinal stromal tumors or soft-tissue sarcoma. Clin Cancer Res 2014;20:3603-12

15. Schöffski P, Sufliarsky J, Gelderblom H, Blay JY, Strauss SJ, Stacchiotti S et al. Crizotinib in patients with advanced, inoperable inflammatory myofibroblastic tumours with and without anaplastic lymphoma kinase gene alterations (European Organisation for Research and Treatment of Cancer 90101 CREATE): a multicentre, single-drug, prospective, non-randomised phase 2 trial. Lancet Respir Med 2018;6:43141

16. Schöffski P, Wozniak A, Kasper B, Aamdal S, Leahy MG, Rutkowski P et al. Activity and safety of crizotinib in patients with alveolar soft part sarcoma with rearrangement of TFE3: European Organization for Research and Treatment of Cancer (EORTC) phase II trial 90101 'CREATE'. Ann Oncol 2018;29:758-65

17. Schöffski P, Wozniak A, Stacchiotti S, Rutkowski P, Blay JY, Lindner LH et al. Activity and safety of crizotinib in patients with advanced clear-cell sarcoma with MET alterations: European Organization for Research and Treatment of Cancer phase II trial 90101 'CREATE'. Ann Oncol 2017;28:3000-8

18. Kopetz S, Lemos R, Powis G. The promise of patient-derived xenografts: the best laid plans of mice and men. Clin Cancer Res 2012;18:5160-2

19. Pan CX, Zhang H, Tepper CG, Lin TY, Davis RR, Keck J et al. Development and characterization of bladder cancer patient-derived xenografts for molecularly guided targeted therapy. PLoS One 2015;10:e0134346

20. Nemati F, Sastre-Garau X, Laurent C, Couturier J, Mariani P, Desjardins L et al. Establishment and characterization of a panel of human uveal melanoma xenografts derived from primary and/or metastatic tumors. Clin Cancer Res 2010;16:2352-62 
21. Dong X, Guan J, English JC, Flint J, Yee J, Evans K et al. Patient-derived first generation xenografts of non-small cell lung cancers: promising tools for predicting drug responses for personalized chemotherapy. Clin Cancer Res 2010;16:1442-51

22. Burgenske DM, Monsma DJ, Dylewski D, Scott SB, Sayfie AD, Kim Dg et al. Establishment of genetically diverse patient-derived xenografts of colorectal cancer. Am J Cancer Res 2014;4:82437

23. Dodd RD, Mito JK, Kirsch DG. Animal models of soft-tissue sarcoma. Dis Model Mech 2010;3:55766

24. Cornillie J, Wozniak A, Pokreisz P, Casazza A, Vreys L, Wellens J et al. In vivo antitumoral efficacy of PhAc-ALGP-doxorubicin, an enzyme-activated doxorubicin prodrug, in patient-derived soft tissue sarcoma xenograft models. Mol Cancer Ther 2017;16:1566-75.

25. McGraw KO, Wong SP. Forming inferences about some intraclass correlation coefficients. Psychol Methods 1996;1:30-46

26. Floris G, Wozniak A, Sciot R, Li H, Friedman L, Van Looy T et al. A potent combination of the novel PI3K Inhibitor, GDC-0941, with imatinib in gastrointestinal stromal tumor xenografts: long-lasting responses after treatment withdrawal. Clin Cancer Res 2013;19:620-30

27. Van Looy T, Wozniak A, Floris G, Sciot R, Li H, Wellens J et al. Phosphoinositide 3-kinase inhibitors combined with imatinib in patient-derived xenograft models of gastrointestinal stromal tumors: rationale and efficacy. Clin Cancer Res 2014;20:6071-82

28. Gebreyohannes YK, Schöffski P, Van Looy T, Wellens J, Vreys L, Cornillie J et al. Cabozantinib Is Active against Human Gastrointestinal Stromal Tumor Xenografts Carrying Different KIT Mutations. Mol Cancer Ther 2016;15:2845-52

29. Ben-David U, Ha G, Tseng Y-Y, Greenwald NF, Oh C, Shih J, et al. Patient-derived xenografts undergo mouse-specific tumor evolution. Nature Genet 2017;49:1567-75

30. Frapoli R, Tamborini E, Virdis E, Bello E, Tarantino E, Marchini S et al. Novel models of myxoid liposarcoma xenografts mimicking the biological and pharmacological features of human tumors. Clin Cancer Res 2010;16:4958-67

31. Cuppens T, Depreeuw J, Annibali D, Thomas D, Hermans E, Gommé E et al. Establishment and characterization of uterine sarcoma and carcinosarcoma patient-derived xenografts. Gynecol Oncol 2017 Jun 16;doi:10.1016/j.ygyno.2017.06.005 [Epub ahead of print]

32. Castellsagué J, Gel B, Fernández-Rodríguez J, Llatjós R, Blanco I, Benavente Y et al. Comprehensive establishment and characterization of orthoxenograft mouse models of malignant peripheral nerve sheath tumors for personalized medicine. EMBO Mol Med 2015;7:608-27

33. Stacchiotti S, Saponara M, Frapolli R, Tortoreto M, Cominetti D, Provenzano S et al. Patientderived solitary fibrous tumour xenografts predict high sensitivity to doxorubicin/dacarbazine combination confirmed in the clinic and highlight the potential effectiveness of trabectedin or eribulin against this tumour. Eur J Cancer 2017;76:84-92

34. Igarashi K, Kawaguchi K, Murakami T, Kiyuna T, Miyake K, Yamamoto N et al. A novel anionicphosphate-platinum complex effectively targets an undifferentiated pleomorphic sarcoma better than cisplatinum and doxorubicin in a patient-derived orthotopic xenograft (PDOX). Oncotarget 2017 Jun 28;doi:10.18632/Oncotarget.18806 [Epub ahead of print]

35. Bharathy N, Svalina MN, Settelmeyer TP, Cleary MM, Berlow NE, Airhart SD et al. Preclinical testing of the glycogen synthase kinase- $3 \beta$ inhibitor tideglusib for rhabdomyosarcoma. Oncotarget 2017 Jun 16;doi:10.18632/Oncotarget.18520 [Epub ahead of print]

36. Oyama R, Takahashi M, Yoshida A, Sakumoto M, Takai Y, Kito F et al. Generation of novel patientderived CIC-DUX4 sarcoma xenografts and cell lines. Sci Rep 2017;7:4712;doi:10.1038/s41598017-04967-0

37. Ray-Coquard I, Montesco MC, Coindre JM, Dei Tos AP, Lurkin A, Ranchère-Vince D et al. Sarcoma: concordance between initial diagnosis and centralized expert review in a population-based study within three European regions. Ann Oncol 2012;23:2442-9

38. Stebbing J, Paz K, Schwartz GK, Wexler LH, Maki R, Pollock RE et al. Patient-derived xenografts for individualized care in advanced sarcoma. Cancer 2014;120:2006-15

39. Roife D, Kang Y, Wang L, Fang B, Swisher SG, Gershenwald JE et al. Generation of patient-derived xenografts from fine needle aspirates or core needle biopsy. Surgery 2017;161:1246-54 
40. Trojani M, Contesso G, Coindre JM, Rouesse J, Bui NB, de Mascarel A et al. Soft-tissue sarcomas of adults; study of pathological prognostic variables and definition of a histopathological grading system. Int J Cancer 1984;33:37-42

41. Hidalgo M, Amant F, Biankin AV, Budinská E, Byrne AT, Caldas C et al. Patient-derived xenograft models: an emerging platform for translational cancer research. Cancer Discov 2014;4:998-1013

42. Ding L, Ellis MJ, Li S, Larson DE, Chen K et al. Genome remodelling in a basal-like breast cancer metastasis and xenograft. Nature 2010;464:999-1005

43. Kresse SH, Meza-Zepeda LA, Machado I, Llombart-Bosch A, Myklebost O. Preclinical xenograft models of human sarcoma show nonrandom loss of aberrations. Cancer 2012;118:558-70

44. Zhan B, Wen S, Lu J, Shen G, Lin X, Feng J et al. Identification and causes of metabonomic difference between orthotopic and subcutaneous xenograft of pancreatic cancer. Oncotarget 2017;8:61264-81

45. Alkema NG, Tomar T, Duiker EW, Meersma GJ, Klip H, van der Zee AG et al. Biobanking of patient and patient-derived xenograft ovarian tumour tissue: efficient preservation with low and high fetal calf serum based methods. Sci Rep 2015;5:14495

46. Izumchenko E, Paz K, Ciznadija D, Sloma I, Katz A, Vasquez-Dunddel D et al. Patient-derived xenografts effectively capture responses to oncology therapy in a heterogeneous cohort of patients with solid tumors. Ann Oncol 2017;28:2595-605

47. Li H, Wozniak A, Sciot R, Cornillie J, Wellens J, Van Looy T et al. Pazopanib, a receptor tyrosine kinase inhibitor, suppresses tumor growth through angiogenesis in dedifferentiated liposarcoma xenograft models. Transl Oncol 2014;7:665-71 
Table 1 Characteristics of donor patients and xenografted tissue $(\mathrm{N}=188)$ with the corresponding in vivo engraftment rate (\%).

\begin{tabular}{|c|c|c|c|}
\hline Characteristic & Patients [n] & $\begin{array}{c}\text { Successful } \\
\text { engraftment }\end{array}$ & P-value \\
\hline \multicolumn{4}{|l|}{ Sex } \\
\hline Male & $104(55 \%)$ & $33 \%$ & \multirow{2}{*}{0.876} \\
\hline Female & $84(45 \%)$ & $32 \%$ & \\
\hline \multicolumn{4}{|l|}{ Age at diagnosis (years) } \\
\hline$<60$ & 99 (53\%) & $31 \%$ & \multirow{2}{*}{0.757} \\
\hline$\geq 60$ & 89 (47\%) & $34 \%$ & \\
\hline \multicolumn{4}{|c|}{ Origin of xenografted sample } \\
\hline Biopsy & $52(28 \%)$ & $22 \%$ & \multirow{2}{*}{0.215} \\
\hline Surgery & $136(72 \%)$ & $34 \%$ & \\
\hline Primary tumor & $129(68 \%)$ & $34 \%$ & \multirow{2}{*}{0.707} \\
\hline Local relapse & 35 (19\%) & $31 \%$ & \\
\hline \multicolumn{4}{|c|}{ Anatomical location sample } \\
\hline Extremities & $88(47 \%)$ & $36 \%$ & \multirow{6}{*}{0.504} \\
\hline Abdomen & $45(24 \%)$ & $29 \%$ & \\
\hline Trunk & $31(16 \%)$ & $35 \%$ & \\
\hline Pelvis & $22(12 \%)$ & $18 \%$ & \\
\hline Head and neck region & $2(1 \%)$ & $50 \%$ & \\
\hline Metastatic lesion & $24(13 \%)$ & $25 \%$ & \\
\hline \multicolumn{4}{|c|}{ Local relapse at any time during the course of disease } \\
\hline No & $141(75 \%)$ & $33 \%$ & \multirow{2}{*}{0.721} \\
\hline Yes & $47(25 \%)$ & $30 \%$ & \\
\hline \multicolumn{4}{|c|}{ Metastatic disease at any time during the course of disease } \\
\hline No & $111(59 \%)$ & $27 \%$ & \multirow{2}{*}{0.059} \\
\hline Yes & 77 (41\%) & $40 \%$ & \\
\hline
\end{tabular}


Table 2 Histopathological subtypes of xenografted tissue $(\mathrm{N}=188)$ with the corresponding in vivo engraftment rate (\%) and number of established models.

\begin{tabular}{|c|c|c|c|c|}
\hline \multirow{2}{*}{$\begin{array}{l}\text { Histology of xenografted tissue } \\
\text { Dedifferentiated liposarcoma }\end{array}$} & \multicolumn{2}{|c|}{ Patients [n] } & \multirow{2}{*}{$\begin{array}{c}\begin{array}{c}\text { Successful } \\
\text { engraftment }\end{array} \\
32 \%\end{array}$} & \multirow{2}{*}{$\begin{array}{c}\begin{array}{c}\text { Established } \\
\text { models [n] }\end{array} \\
5\end{array}$} \\
\hline & 31 & $(16.49 \%)$ & & \\
\hline Leiomyosarcoma & 27 & $(14.36 \%)$ & $41 \%$ & 5 \\
\hline Myxofibrosarcoma & 21 & $(11.17 \%)$ & $43 \%$ & 7 \\
\hline Myxoid liposarcoma & 13 & $(6.91 \%)$ & $15 \%$ & - \\
\hline Atypical lipomatous tumor & 10 & $(5.32 \%)$ & $20 \%$ & - \\
\hline Undifferentiated pleomorphic sarcoma & 9 & $(4.79 \%)$ & $33 \%$ & 3 \\
\hline Synovial sarcoma & 8 & $(4.26 \%)$ & $38 \%$ & 1 \\
\hline Undifferentiated sarcoma, not otherwise specified & 8 & $(4.26 \%)$ & $25 \%$ & 1 \\
\hline Malignant peripheral nerve sheath tumor & 6 & (3.19\%) & $67 \%$ & 2 \\
\hline Solitary fibrous tumor & 6 & (3.19\%) & $17 \%$ & - \\
\hline Undifferentiated spindle cell sarcoma & 6 & (3.19\%) & $33 \%$ & - \\
\hline Angiosarcoma & 5 & $(2.66 \%)$ & $0 \%$ & - \\
\hline Extraskeletal myxoid chondrosarcoma & 5 & $(2.66 \%)$ & $100 \%$ & - \\
\hline Other rare subtypes: & 33 & $(17.55 \%)$ & $27 \%$ & 8 \\
\hline Pleomorphic liposarcoma & 4 & & & - \\
\hline Undifferentiated radiation-associated STS & 3 & & & - \\
\hline Epithelioid sarcoma & 3 & & & - \\
\hline Telangiectatic extraskeletal osteosarcoma & 2 & & & 1 \\
\hline Inflammatory myofibroblastic tumor & 2 & & & - \\
\hline Alveolar rhabdomyosarcoma & 2 & & & - \\
\hline Chondrosarcoma & 2 & & & - \\
\hline Pulmonary artery intimal sarcoma & 2 & & & 1 \\
\hline CIC-rearranged round cell sarcoma & 1 & & & 1 \\
\hline Dermatofibrosarcoma protuberans & 1 & & & - \\
\hline Desmoplastic small round cell tumor & 1 & & & - \\
\hline Epithelioid hemangioendothelioma & 1 & & & 1 \\
\hline Ewing sarcoma & 1 & & & - \\
\hline Low-grade fibromyxoid sarcoma & 1 & & & - \\
\hline Mesenchymal chondrosarcoma & 1 & & & 1 \\
\hline Pleomorphic rhabdomyosarcoma & 1 & & & 1 \\
\hline Rhabdomyosarcoma, not otherwise specified & 1 & & & 1 \\
\hline Sclerosing epithelioid fibrosarcoma & 1 & & & - \\
\hline Myxoinflammatory fibroblastic sarcoma & 1 & & & 1 \\
\hline Well-differentiated spindle cell liposarcoma & 1 & & & - \\
\hline Sarcoma ex teratoma & 1 & & & - \\
\hline
\end{tabular}




\section{Figure Legends}

Figure 1. Survival according to engraftment or establishment of PDX model.

Survival of patients according to (A) successful engraftment of donor tissue and (B) successful model establishment in NMRI nu/nu immunodeficient mice

PDX: patient-derived xenograft

Figure 2. PDX maintain the histological features of the original donor tumor throughout passaging.

Representative images of hematoxylin and eosin-stained sections of donor tumors and the last available passage of the corresponding STS PDX model $(n=24)$. Images were captured under 200-fold magnification. UZLX-STS41 was not included, as paraffin-embedded patient material from this model was not available.

DDLPS: dedifferentiated liposarcoma; MPNST: malignant peripheral nerve sheath tumor; p.: passage; PDX: patient-derived xenograft

Figure 3. Xenografted and original patient tumors show similar patterns of expression of characteristic immunohistochemical markers.

Examples of representative immunostainings of original patient tissue and different passages of the corresponding PDX model, showing stable immunohistochemical profile throughout passaging. Images were captured under 200 -fold magnification.

$\alpha$-SMA: alpha smooth muscle actin; MDM2: mouse double minute 2 homolog; p.: passage

Figure 4. Subtype-specific genetic changes are preserved in PDX models.

FISH analysis showing (A) MDM2 amplification in UZLX-STS5p.12 and (B) MDM2 amplification in UZLX-STS112p.4 [for both: dual-color FISH probe set, targeting MDM2 (red) and CEP12 (green)], (C) SS18 rearrangement in UZLX-STS7p.1 [dual-color FISH probe set, targeting distal (green) and proximal (red) region to SS18] (D) CAMTA1 rearrangement in UZLX-STS15p.10 [dual-color FISH probe set, targeting distal (green) and proximal (red) region to CAMTA1], (E) CIC unbalanced rearrangement in UZLX-STS134p.2 [dual-color FISH probe set, targeting distal (green) and proximal (red) region to $C I C$ ] and (F) HEY1-NCOA2 fusion in UZLX-STS41p.12 [dualcolor FISH probe set, targeting HEY1 (red) and NCOA2 (green) regions]. Dashed arrows show split signals, full arrows show fusion signals.

CAMTA1: calmodulin binding transcription activator 1; CIC: capicua transcriptional repressor; DDLPS: dedifferentiated liposarcoma; FISH: fluorescence in situ hybridization; HEY1: hes-related family bHLH transcription factor with YRPW motif 1; MDM2: mouse double minute 2 homolog; NCOA2: nuclear receptor coactivator 2; p.: passage; SS18: synovial sarcoma translocation, chromosome 18 


\section{Cornillie et al. Figure 1}
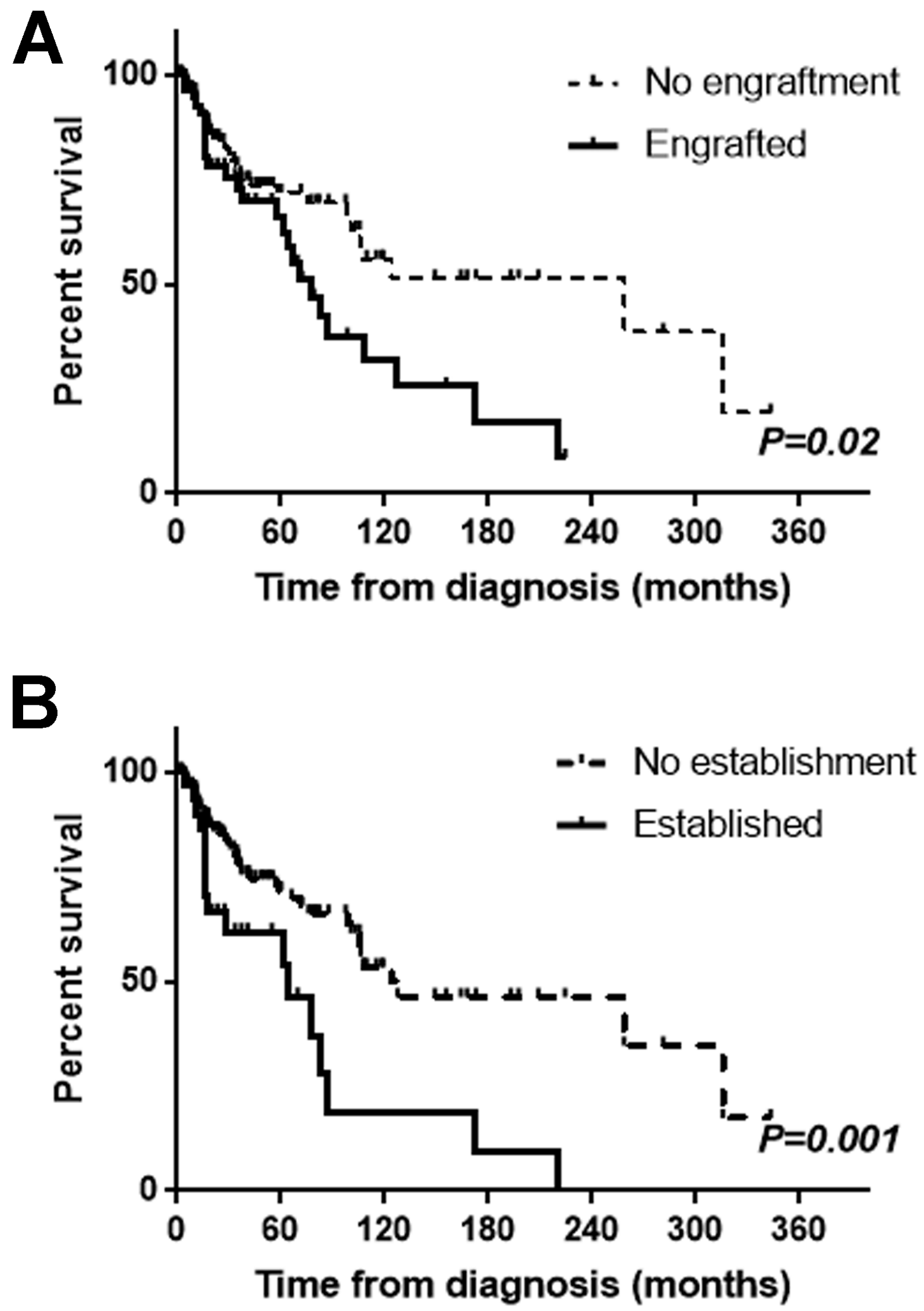


\section{Cornillie et al. Figure 2}

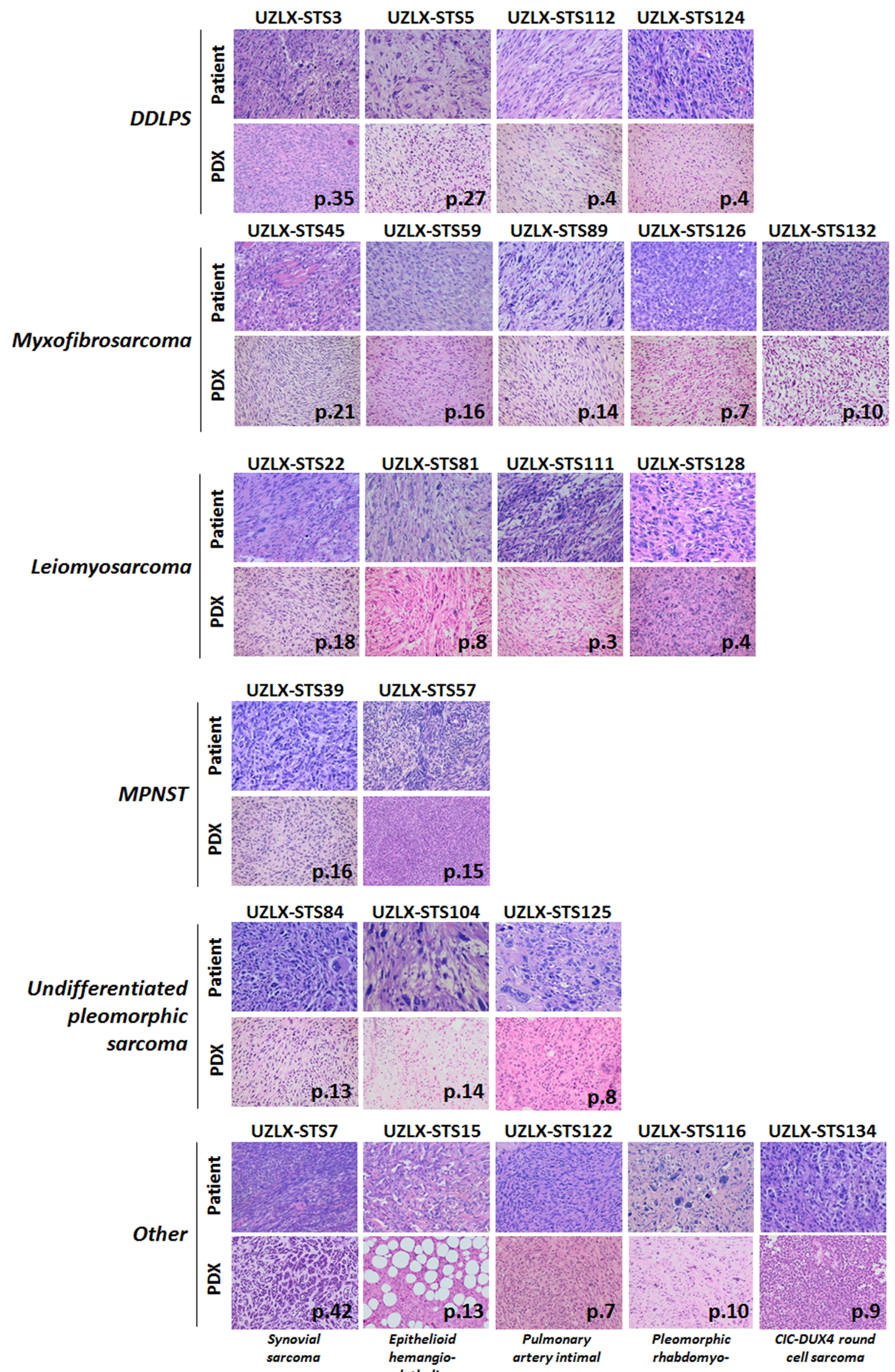

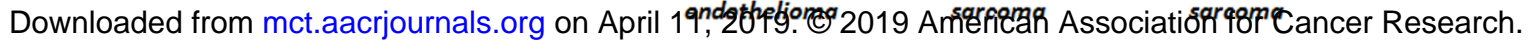




\section{Cornillie et al. Figure 3}

\section{UZLX-STS3}

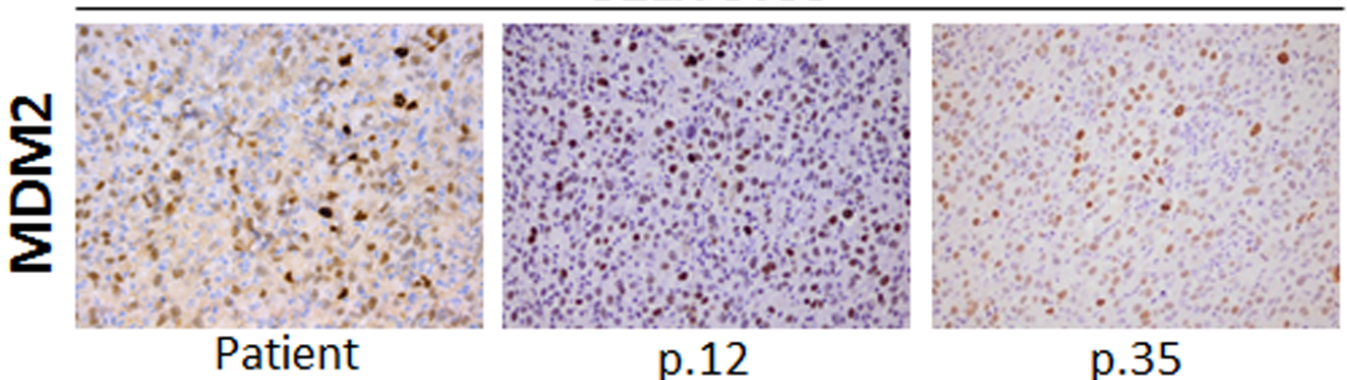

\section{UZLX-STS128}

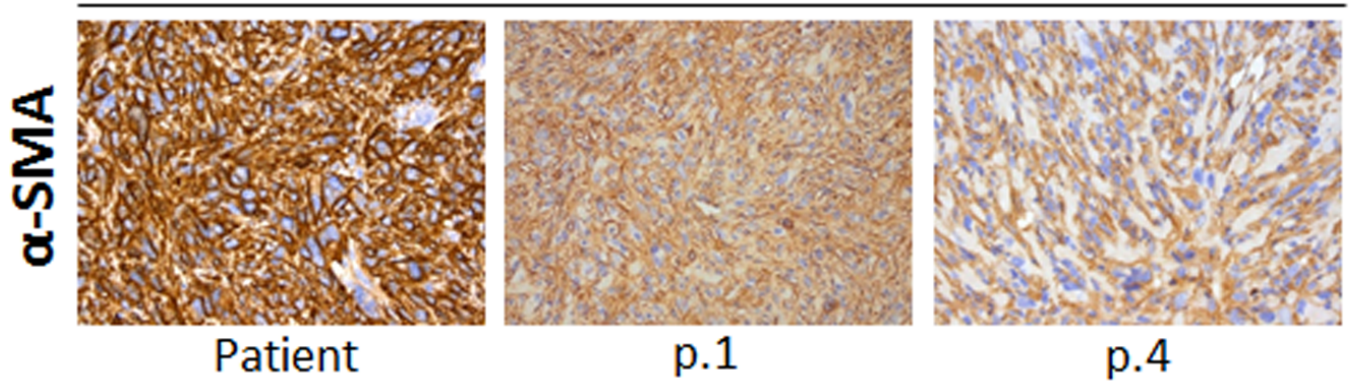

\section{UZLX-STS81}

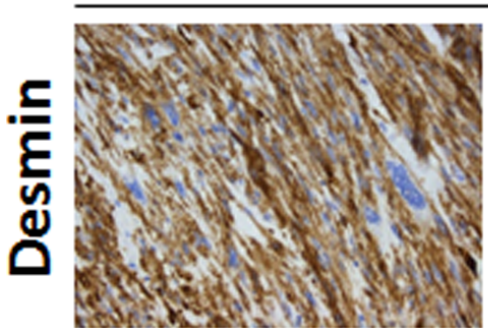

Patient

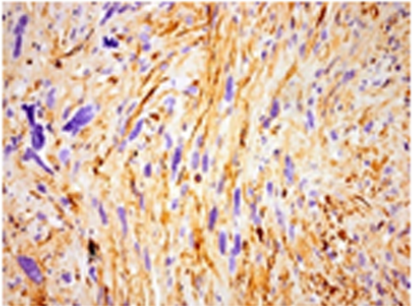

p. 2

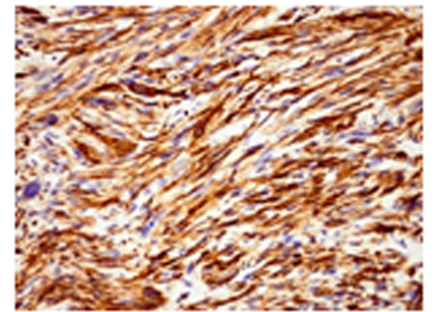

p. 8

\section{UZLX-STS39}

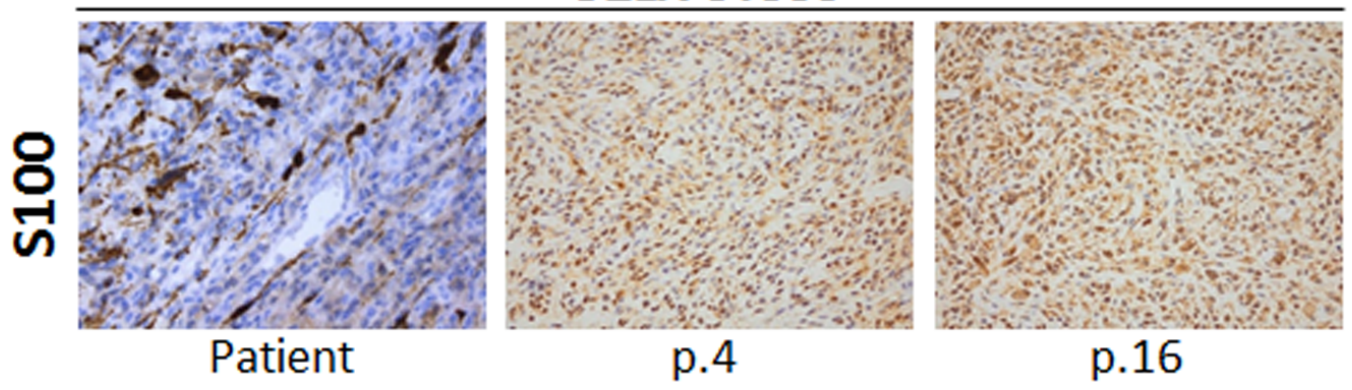

\section{UZLX-STS116}

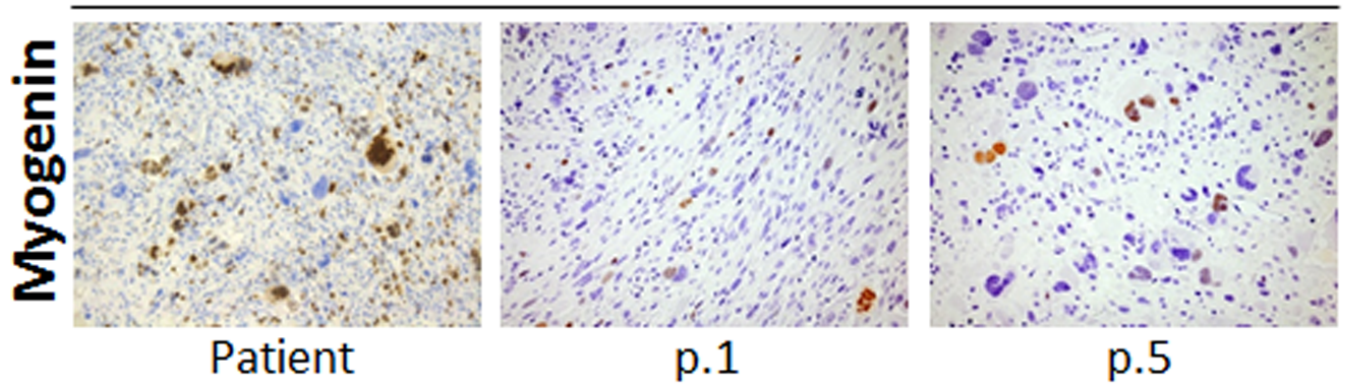




\section{Cornillie et al. Figure 4}
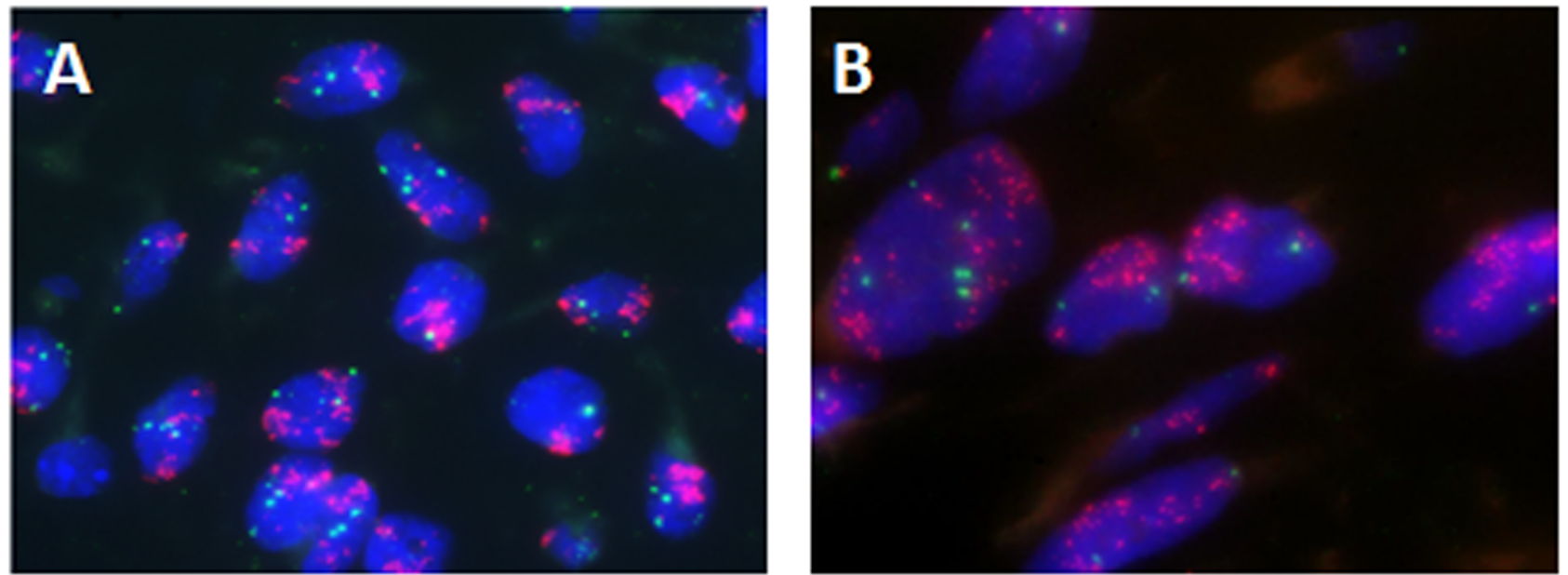

MDM2 amplification

MDM2 amplification

UZLX-STS5p.12 (DDLPS)

UZLX-STS112p.4 (DDLPS)

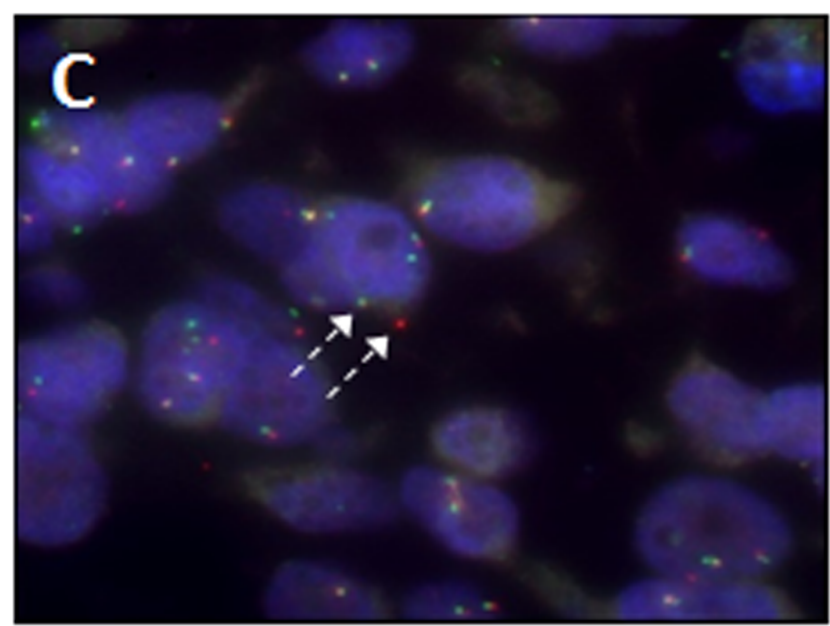

SS18 rearrangement

UZLX-STS7p.1

(synovialsarcoma)

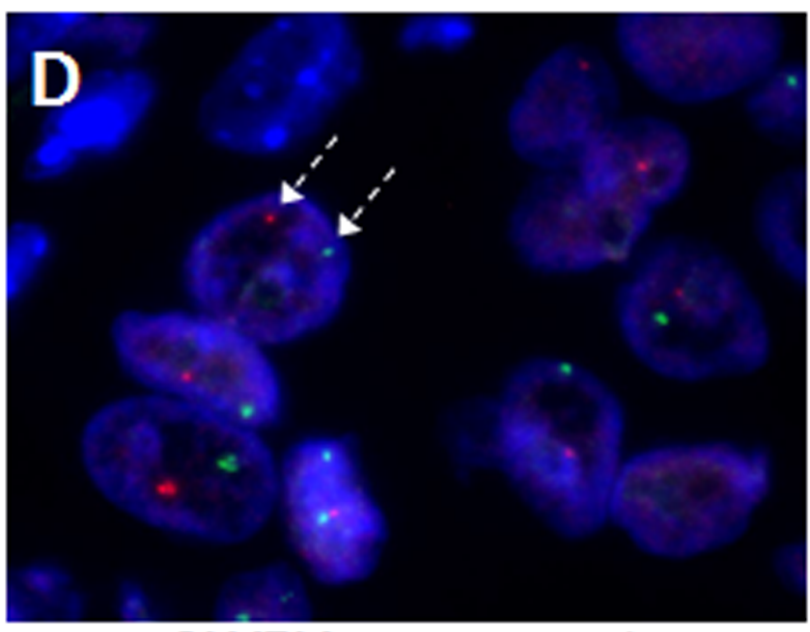

CAMTA1 rearrangement

UZLX-STS15p.10

(epithelioid hemangioendothelioma)

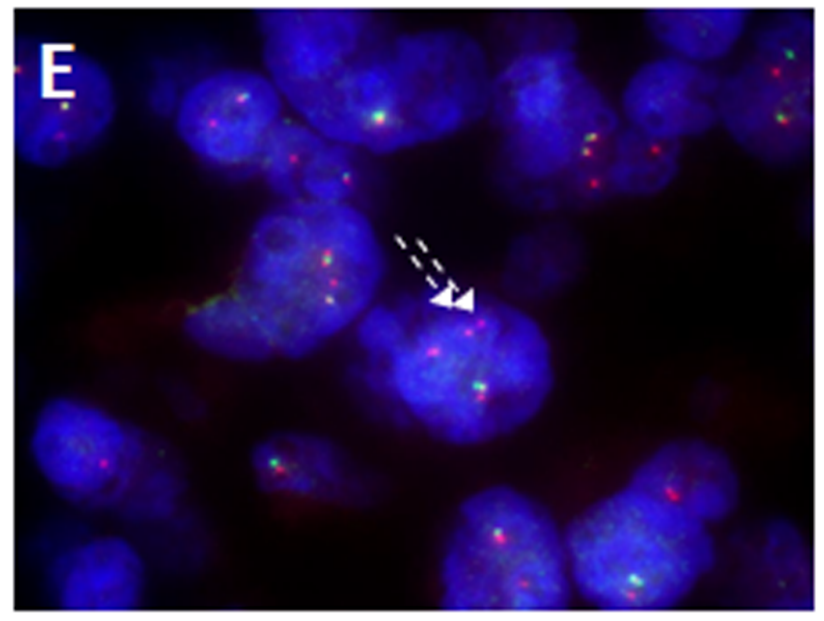

$\mathrm{ClC}$ unbalanced rearrangement

$$
\text { UZLX-STS134p. } 2
$$

(CIC-DUX4 fusion-positive Ewing-like

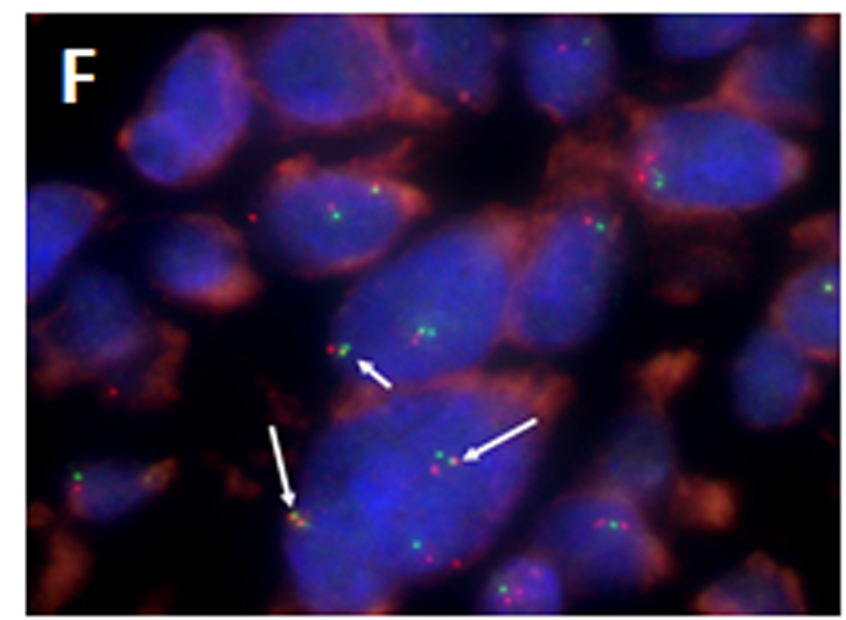

HEY1-NCOA2 fusion

UZLX-STS 41 p. 12

(mesenchymal chondrosarcoma) 


\section{Molecular Cancer Therapeutics}

\section{Establishment and characterization of histologically and molecularly stable soft tissue sarcoma xenograft models for biological studies and preclinical drug testing}

Jasmien Cornillie, Agnieszka Wozniak, Haifu Li, et al.

Mol Cancer Ther Published OnlineFirst April 8, 2019.

\begin{tabular}{|rl|}
\hline Updated version & $\begin{array}{l}\text { Access the most recent version of this article at: } \\
\text { doi:10.1158/1535-7163.MCT-18-1045 }\end{array}$ \\
$\begin{array}{r}\text { Supplementary } \\
\text { Material }\end{array}$ & $\begin{array}{l}\text { Access the most recent supplemental material at: } \\
\text { http://mct.aacrjournals.org/content/suppl/2019/04/06/1535-7163.MCT-18-1045.DC1 }\end{array}$ \\
$\begin{array}{r}\text { Author } \\
\text { Manuscript }\end{array}$ & $\begin{array}{l}\text { Author manuscripts have been peer reviewed and accepted for publication but have not yet } \\
\text { been edited. }\end{array}$ \\
\hline
\end{tabular}

E-mail alerts Sign up to receive free email-alerts related to this article or journal.

Reprints and To order reprints of this article or to subscribe to the journal, contact the AACR Publications Subscriptions Department at pubs@aacr.org.

Permissions To request permission to re-use all or part of this article, use this link http://mct.aacrjournals.org/content/early/2019/04/06/1535-7163.MCT-18-1045.

Click on "Request Permissions" which will take you to the Copyright Clearance Center's (CCC) Rightslink site. 